\title{
Experimental Investigation of Mode Shapes of Symmetric Structures
}

\author{
Róbert Huňady * and Martin Hagara
}

Technical University of Košice, Faculty of Mechanical Engineering, Department of Applied Mechanics and Mechanical Engineering, Letná 9, 04200 Košice, Slovakia

\begin{abstract}
Paper deals with experimental determination of modal parameters of symmetric structures which have so-called coupled mode shapes of vibration. These modes usually have almost the same frequencies. This is why it could be difficult to identify them from frequency response functions where their peaks overlap. Main attention is focused on the using of Complex Mode Indicator Function (CMIF) that is able to separate coupled and also closely spaced modes by using singular value decomposition of multiple reference FRF matrix. In the paper there is described a theoretical background of CMIF and its application in spatial domain parameter estimation. Its functionality is explained on an analytical model of mechanical system of six degrees of freedom. Its practical application is presented by experimental modal analysis of circular saw blade.
\end{abstract}

Keywords: Symmetric structure; coupled modes; experimental modal analysis; complex mode indicator function.

\section{Introduction}

It is well known that mode shapes of mass and shape symmetric objects are symmetric but such objects also have mode shapes which are mutually quite the same but rotated about the symmetry axis. Such mode shapes are called coupled and theoretically they should be belonging to same frequency. In practice, there is always some inhomogeneity of a material or the shape of a specimen is not exactly symmetric so the frequency shift of coupled modes occurs. By experimental modal investigation such shift can be also created by inconvenient support of a tested specimen or by application of transducers at improper locations.

There exist several algorithms that allow to estimate modal parameters either in time or frequency domain, but not every is able to distinguish coupled or too closed modes. The Complex Mode Indicator Function is very popular numerical tool commonly used in experimental modal analysis for the estimation of modal parameters $[1,2,3]$. The CMIF identifies modes by showing the physical magnitude of each mode and the damped natural frequency for each root. Since multiple reference data is applied in CMIF, repeated roots can be detected. The CMIF also gives global modal parameters, such as damped natural frequencies, mode shapes and modal participation vectors $[4,5,6]$.

\section{Objective and Scope of Research}

The dynamic behavior of mechanical structure can be described by Frequency 
Response Functions (FRF), each of which defines the relationship between one input and one output, i.e. between excitation and response [7]. The set of these functions forms so-called FRF matrix $[H(j \omega)]$ that represents a response model of given structure. In the modal analysis area, by assuming linear and time invariant systems, the FRF matrix of a $N$ degree-of-freedom system can be expressed as

$$
\begin{aligned}
& {[H(j \omega)]=\sum_{r=1}^{2 N} \frac{\left[R_{r}\right]}{j \omega-\lambda_{r}}=} \\
& =\sum_{r=1}^{2 N}\{\phi\}_{r} \frac{Q_{r}}{j \omega-\lambda_{r}}\{L\}_{r}^{H}
\end{aligned}
$$

or in matrix form

$$
[H(j \omega)]=[\Phi]\left[\frac{Q_{r}}{j \omega-\lambda_{r}}\right][L]
$$

where: $\left[R_{r}\right]$ is the $r^{\text {th }}$ residue matrix, $\{\phi\}_{r}$ is the $r^{\text {th }}$ mode shape, $\{L\}_{r}$ is the $r^{\text {th }}$ modal participation vector, $[\Phi]$ is mode shape matrix, $[L]$ is modal participation factor matrix, $Q_{r}$ is the scaling factor of $r^{\text {th }}$ mode, $\lambda_{r}$ is the complex pole of $r^{\text {th }}$ mode.

By taking the singular value decomposition of the FRF matrix at each spectral line $[4,8]$, we obtain an expression equivalent to Eq. (2)

$$
[H(j \omega)]=[U][S][V]^{H}
$$

where: $[U]$ is left singular matrix, $[V]$ is right singular matrix, $[S]$ is diagonal matrix of singular values.

Matrices $[U]$ and $[V]$ are unitary and have orthogonal columns. Mode shapes are represented by left singular vectors of $[U]$ matrix and modal scale factors are represented by right singular vectors of $[V]$ matrix.

The comparison of Eq. (2) and Eq. (3) shows that singular values are proportional to the scaling factor divided by the difference between the discrete frequency and the modal frequency. For a given mode, since the scaling factor is a constant, the closer the modal frequency is to the discrete (measured) frequency, the larger the singular value will be. The Complex Mode Indication Function is defined as the eigenvalues solved from the normal matrix, which is formed from the FRF matrix, i.e. $[H(j \omega)]^{H}[H(j \omega)]$. By this definition, the CMIF is equal to the square of the magnitude of the singular value $[4,5]$

$$
\begin{aligned}
& \operatorname{CMIF}_{k}(j \omega)=\mu_{k}(j \omega)=\sigma_{k}^{2}(j \omega) \\
& k=1,2, \ldots, N
\end{aligned}
$$

where: $C M I F_{k}(j \omega)$ is the $k^{\text {th }}$ singular curve, $\mu_{k}(j \omega)$ is the $k^{\text {th }}$ eigenvalue of the normal matrix of FRF matrix, $\sigma_{k}(j \omega)$ is the $k^{\text {th }}$ singular value of the FRF matrix, $N_{r}$ is the number of dominant modes that contribute to the final response of the structure.

The CMIF plot is the plot of singular values on a log magnitude as a function of frequency. The peaks detected in the CMIF plot indicate the existence of modes, and the located frequencies give the corresponding damped natural frequencies. However, it is necessary to note that not all peaks of CMIF indicate the modes. Some peaks are generated as a consequence of noise, leakage or nonlinearity. A cross (singular value) effect can also generate an apparent peak. The cross effect occurs due to way the CMIF is plotted and can be observed especially on the lower singular curves because the largest singular curve is plotted first. The indication of coupled modes is given by presence of more peaks of CMIFs at a same frequency.

Left singular vectors correspond to scaled mode shape vector. Unscaled mode shape can be solved as [1]

$$
\begin{aligned}
& \left\{u\left(j \omega_{p}\right)\right\}_{k}=\left[H\left(j \omega_{p}\right)\right]\left\{v\left(j \omega_{p}\right)\right\}_{k} \mu\left(j \omega_{p}\right)_{k}^{-1} \\
& k=1,2, \ldots, N_{k}
\end{aligned}
$$

where: $\left\{u\left(j \omega_{p}\right)\right\}_{k}$ is the unscaled mode shape for $k^{\text {th }}$ repeated root at $j \omega_{p},\left\{v\left(j \omega_{p}\right)\right\}_{k}$ is the equivalent mode participation factor for $k^{\text {th }}$ repeated root at $j \omega_{p}, N_{k}$ is the number of repeated roots detected at peak frequency $j \omega_{p}$ that is the approximate damped natural frequency of $r^{\text {th }}$ mode.

\section{Analytical Model}

The functionality of CMIF will be explained on a simple analytical model of linear damped system. Let us consider mechanical system of six degreesof-freedom (Fig. 1), of which physical properties are expressed by following matrices: 


$$
\begin{aligned}
& {[M]=E_{6}} \\
& {[B]=\left[\begin{array}{cccccc}
4 & -2 & 0 & 0 & 0 & 0 \\
-2 & 6 & -4 & 0 & 0 & 0 \\
0 & -4 & 8 & -4 & 0 & 0 \\
0 & 0 & -4 & 8 & -4 & 0 \\
0 & 0 & 0 & -4 & 6 & -2 \\
0 & 0 & 0 & 0 & -2 & 4
\end{array}\right]} \\
& {[K]=10^{4} \cdot\left[\begin{array}{cccccc}
30 & -1 & 0 & 0 & 0 & 0 \\
-1 & 4 & -3 & 0 & 0 & 0 \\
0 & -3 & 10 & -7 & 0 & 0 \\
0 & 0 & -7 & 10 & -3 & 0 \\
0 & 0 & 0 & -3 & 4 & -1 \\
0 & 0 & 0 & 0 & -1 & 30
\end{array}\right]}
\end{aligned}
$$

where $[M],[B],[K]$ are mass matrix, damping matrix and stiffness matrix, respectively.

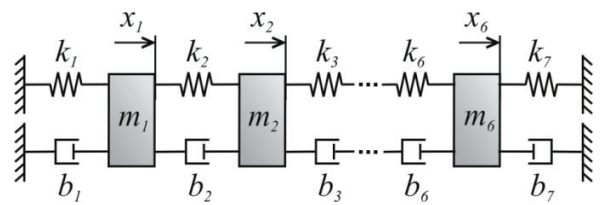

Fig. 1: Model of damped mechanical system of six degrees-offreedom.

For the case of free vibration, the equation of motion of the given system in matrix form is

$$
[M]\{\ddot{x}\}+[B]\{\dot{x}\}+[K]\{x\}=\{0\} .
$$

Frequency response functions representing the result of multiple reference measurement can be calculated from Eq. (1a) after the modal parameters are known. Modal parameters will be taken from eigen solution of state equation where

$$
\{\dot{z}\}=[A]\{z\}
$$

$\{z\}=\left[\begin{array}{l}\{x\} \\ \{\dot{x}\}\end{array}\right]$ is state vector,

$[A]=\left[\begin{array}{cc}{[0]} & {\left[E_{6}\right]} \\ {[M]^{-1}[K]} & {[M]^{-1}[B]}\end{array}\right]$ is state matrix.

The solution leads to eigenvalues $\lambda_{r}$ and eigenvectors $\{\psi\}$ r. The eigenvalues represent complex conjugate poles of the system

$\lambda_{r}=-\delta_{r} \pm j \omega_{d r}$

where: $\delta_{r}=-\operatorname{Re}\left(\lambda_{r}\right)$ is the damping factor of $r^{\text {th }}$ mode, $\omega_{d r}=\operatorname{Im}\left(\lambda_{r}\right)$ is damped natural frequency of $r^{\text {th }}$ mode.

The elements of residue matrices in Eq. (1a) are the product of the modal deformations at the $q^{\text {th }}$ input and $p^{\text {th }}$ response degrees-of-freedom and a modal scaling factor of $r^{\text {th }}$ mode, i.e.

$R_{p q r}=Q_{r} \psi_{p r} \psi_{q r}$

where: $\psi_{p r}$ is the mode shape coefficient at response DOF $p$ of $r^{\text {th }}$ mode, $\psi_{q r}$ is the mode shape coefficient at input DOF $q$ of $r^{\text {th }}$ mode.

For proportionally damped systems, modal scaling factor can be expressed as [5]

$Q_{r}=\frac{1}{j 2 M_{r} \omega_{d r}}$

where: $M_{r}$ is modal mass of $r^{\text {th }}$ mode that can be calculated as

$M_{r}=\{\psi\}_{r}^{T}[M]\{\psi\}_{r}$.

All frequency response functions calculated by this way can be written into the matrix that is FRF matrix of analyzed model. As an example, Fig. 2 shows frequency response functions of the $4^{\text {th }}$ column of FRF matrix.

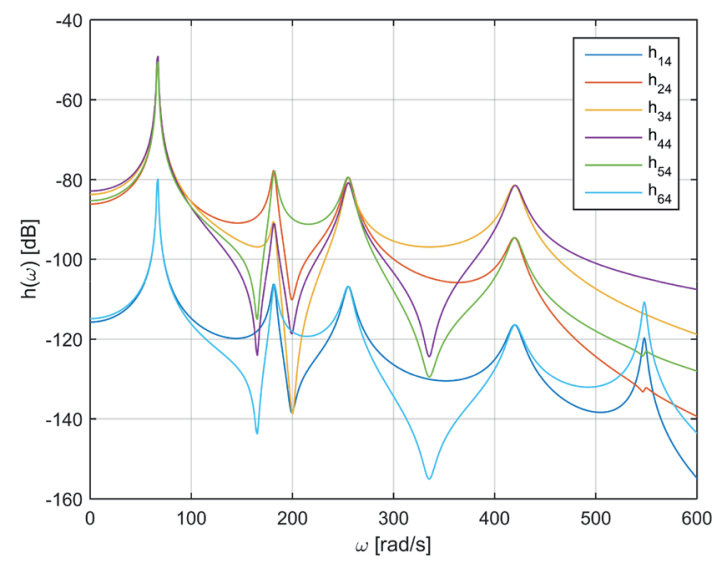

Fig. 2: Example plot of frequency response functions of the given system. 
Now, when the FRF matrix is known, we can proceed to estimation of modal parameters. Complex Mode Indicator Function obtained by singular value decomposition of FRF matrix is plotted in Fig. 3. There are five dominated peaks whose frequencies correspond to the approximate values of natural damped frequencies, but as we can see in Fig. 3 the first and second singular curve overlap each other. In other words they have peaks at the same frequency. It means that these two modes $\left(5^{\text {th }}\right.$ and $\left.6^{\text {th }}\right)$ are coupled. The estimated mode shapes of vibration were extracted from left singular matrix $[U]$ and are shown in Fig. 4. We can observe that one of coupled modes is symmetric and the second one is anti-symmetric.

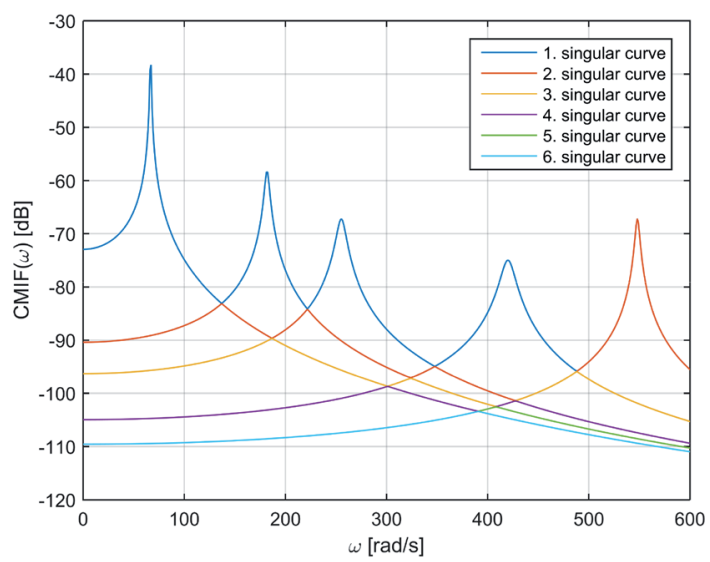

Fig. 3: Complex Mode Indicator Functions of analyzed model.

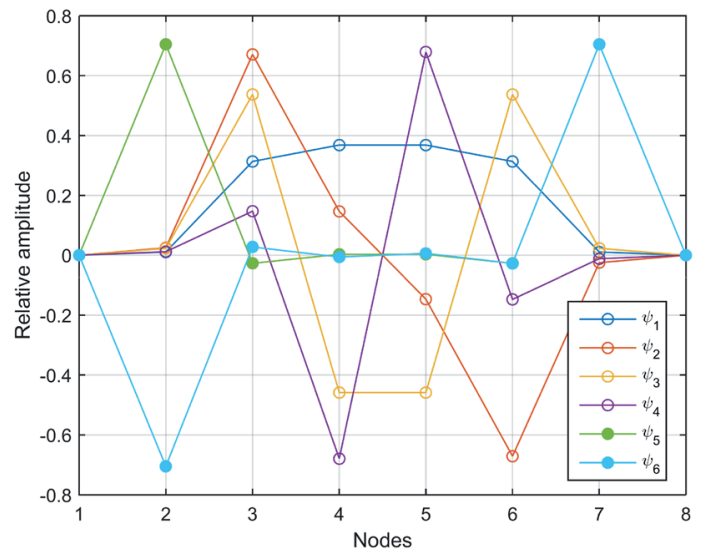

Fig. 4: Scaled mode shapes of analyzed model.

For a comparison, the estimated and also exact modal parameters of given system are presented in Table 1. It should be noted that the accuracy of estimated frequencies and damping ratios depends on resolution of frequency spectrum. Damping ratios noted in Table 1 were estimated from CMIFs by means the half power bandwidth method.

Table 1: Natural frequencies and damping ratios of analyzed system.

\begin{tabular}{|l|l|l|l|l|}
\hline Mode & \multicolumn{2}{c}{ Exact parameters } & \multicolumn{2}{c|}{ Estimated parameters } \\
& $\omega[\mathrm{rad} / \mathrm{s}]$ & $\zeta[\%]$ & \multicolumn{1}{c|}{$\omega[\mathrm{rad} / \mathrm{s}]$} & $\zeta[\%]$ \\
\hline 1 & 66.6650 & 0.6274 & 67.1119 & 0.6385 \\
\hline 2 & 181.7973 & 1.2264 & 181.3022 & 1.2383 \\
\hline 3 & 255.2392 & 1.7638 & 255.4257 & 1.7437 \\
\hline 4 & 420.1205 & 1.5920 & 419.6995 & 1.6071 \\
\hline 5 & 548.0735 & 0.3799 & 547.9132 & 0,3818 \\
\hline 6 & 548.0689 & 0.3795 & 547.9132 & 0,3818 \\
\hline
\end{tabular}

\section{Experimental Case}

For experimental investigation of coupled modes the circular-saw blade with outside diameter of 250 $\mathrm{mm}$ and thickness of $4 \mathrm{~mm}$ was used. Saw blade was supported by elastic very soft foam. There were defined 81 degrees-of-freedom, three of which were reference (Fig. 5).

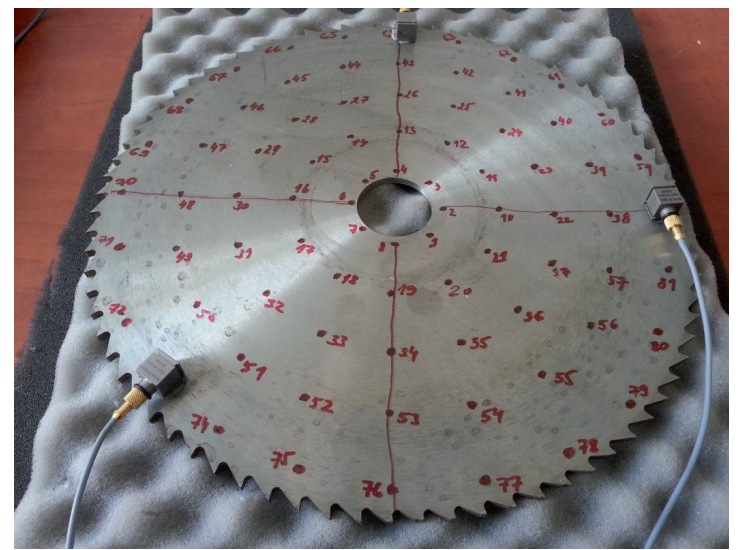

Fig. 5: The tested circular-saw blade.

As a measuring device the Brüel\&Kjaer Pulse system was used. The structure was excited by the impact hammer Brüel\&Kjaer type 8206 with plastic tip. The responses were measured by three uniaxial accelerometers Brüel\&Kjaer type 4507-B. All the measurement was performed in MTC-Hammer software. Span frequency was set at $5000 \mathrm{~Hz}$ and the frequency resolution was $0.7813 \mathrm{~Hz}$. 


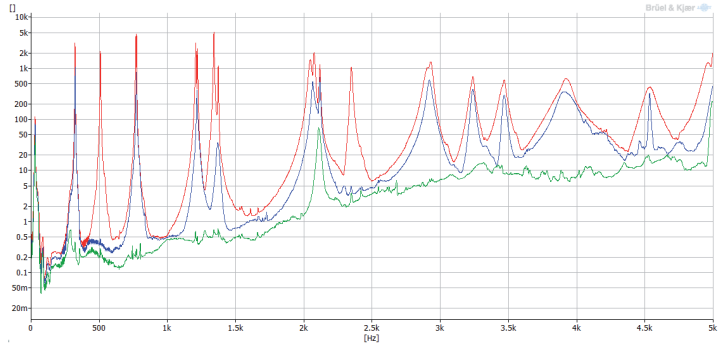

Fig. 6: CMIF functions of exported data.

For next processing the measured FRFs were exported to Pulse Reflex software and CMIF functions were computed (Fig. 6). Modal parameters of the circular-saw blade were determined on the basis of CMIF with using of rational fraction polynomial (RFP) method.

The presence of coupled modes is indicated by the peaks of the same frequency on particular singular curves. We can also observed in Fig. 6 that for second and eighth mode there are no coupled
Table 2: Natural frequencies of circular-saw blade.

\begin{tabular}{|c|c|c|c|c|c|c|c|}
\hline \multicolumn{7}{|c|}{ Frequency of modes [Hz] } \\
1st & 2nd & \multicolumn{1}{c|}{ 3rd } & 4th & \multicolumn{1}{c|}{ 5th } & \multicolumn{1}{c|}{ 6th } & \multicolumn{1}{c|}{ 7th } \\
\hline 321.7 & 507.8 & 767.6 & 1207 & 1340 & 2047 & 2115 \\
323.6 & & 772.8 & 1219 & 1371 & 2075 & 2117 \\
\hline 8th & 9th & 10th & 11th & 12th & 13th & \\
\hline 2347 & 2909 & 3232 & 3457 & 3904 & 4530 & \\
\hline & 2931 & 3239 & 3467 & 3926 & 4534 & \\
\hline
\end{tabular}

mode because only one singular curve has peak on that frequency. The acquired natural frequencies are presented in Table 2.

Frequencies of coupled modes are not exactly same. One reason is an added weight of applied accelerometers that influenced the structural properties of the saw blade and the conditions of its symmetry. For the illustration, only the first seven mode shapes are shown in Table 3.

Table 3: Mode shapes of circular-saw blade.

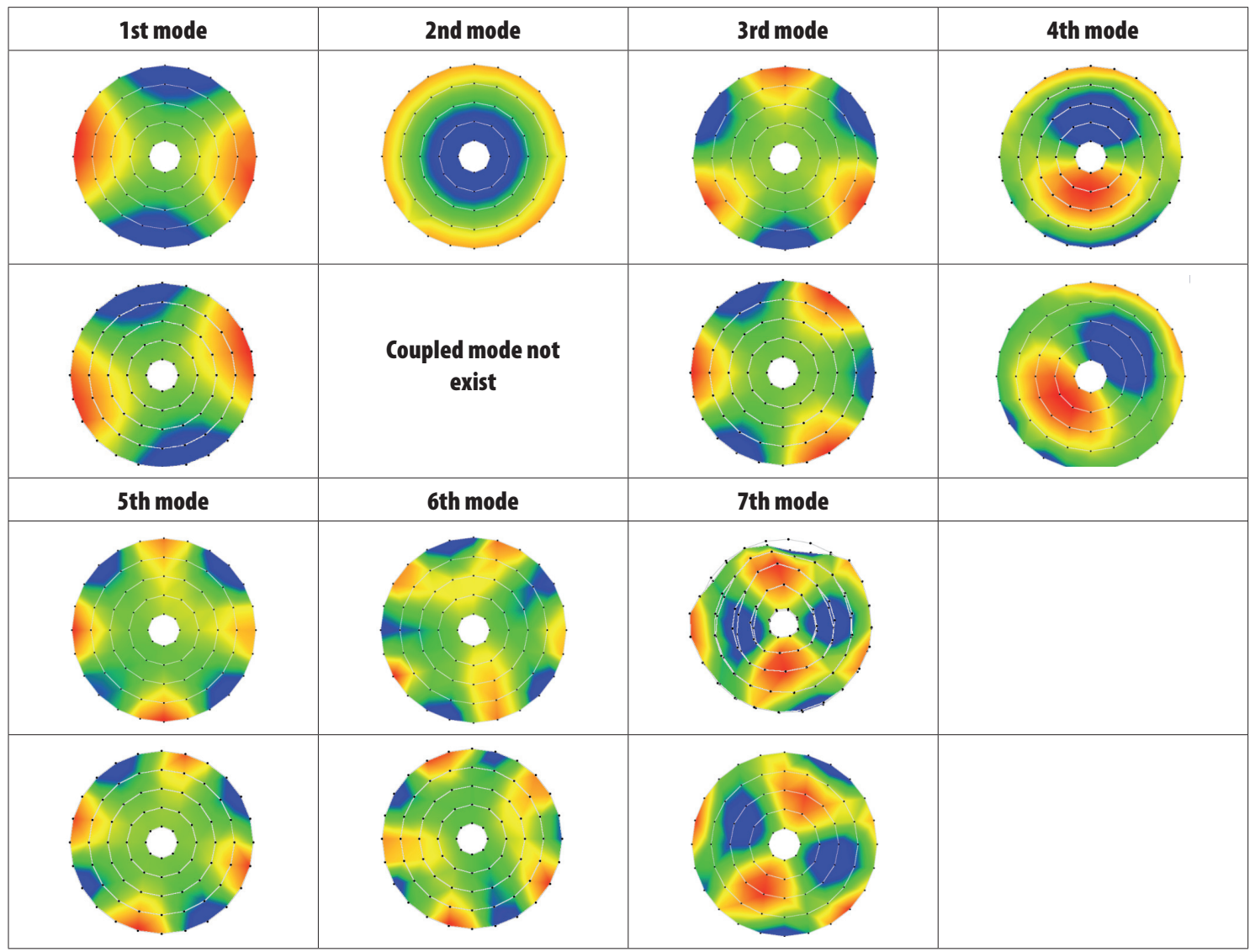




\section{Conclusions}

The estimation of modal parameters is a difficult process because the noise-free measurement has to be performed and proper extraction method has to be used. The paper presented the way of identification of coupled modes by using Complex Mode Indicator Function based on singular value decomposition of FRF matrix. The CMIF appears to be a simple and efficient method for identifying the modes of the complex system. It provides a global estimation of modal parameters, so damped natural frequencies, mode shapes, modal participation vectors and damping ratios are available with relative ease. The accuracy of modal parameters depends on the resolution of frequency spectrum and the conditions of an experiment. As was shown in the paper, transducers applied to a structure can cause a shift of natural frequencies. It is therefore likely that the real natural frequencies of the tested circular-saw blade are higher than the measured values. This phenomenon can be circumvented by using contactless method to measure responses.

\section{Acknowledgments}

The authors would like to thank to project APVV-0091-11 "Využitie metód experimentálneho a numerického modelovania pre zvyšovanie konkurencieschopnosti a inovácie mechanických a mechatronických sústav".

\section{References and Notes}

[1] Trebuňa, F., Huňady, R., Bobovský, Z., Hagara, M.: An Application of High-speed Digital Image Correlation id Determination of Modal Parameters. In: Acta Mechancia Slovaca, Vol. 15, No. 4., 2011, pages 6-13.

[2] Trebuňa, F., Šimčák, F., Huňady, R.: Experimental Analysis of Vibration of Transport Complex for Transportation of Spent Nuclear Fuel. In: Acta Mechancia Slovaca, Vol. 16, No. 3. 2012, pages 26-32.

[3] Trebuňa, F., Huňady, R., Pástor, M., Binda, M.: Analysis of Causes Unwanted Dynamical Phenomenon on Output Pipes of Turbocompressor. In: Acta Mechancia Slovaca, Vol. 15, No. 3., 2011, pages 6-13.

[4] Philips, A.W., Allemang, R.J., Fladung, W.A. (1998) The Complex Mode Indicator Function (CMIF) as a Parameter Estimation Method. 16th International Modal Analysis Conference, IMAC XVI, 6 pp.

[5] Allemang, R.J., Brown, D.L. (2002) Chapter 21: Experimental Modal Analysis. In: Harris, C.M., Piersol, A.G., Harris' Shock and Vibration Handbook, McGraw-Hill Inc., 1568 pp.

[6] Allemang, R.J., Brown, D.L. (2006) A complete review of the complex mode indicator function with applications. International Conference on Noise and Vibration Engineering, ISMA, 38 pp.

[7] Trebuňa, F., Šimčák, F., Huňady, R. (2012) Vibration and Modal Analysis of Mechanical Systems (in Slovak), TU v Košiciach, Košice, $236 \mathrm{pp}$.

[8] Ewins, D.J. (2000) Modal testing: theory, practice and application. Second edition. Wiley, $562 \mathrm{pp}$

\section{Biographical notes}

Róbert Huňady, doc. Ing. Ph.D., (born in 1981) is an associated professor of applied mechanics. Since 2010 he has been working at the Department of Applied Mechanics and Mechanical Engineering, Technical University of Košice, Slovakia. In 2009, he attended EC Marie Curie Training Program SIMVIA2 at Czech Technical University of Prague, Czech republic. His professional activity is mainly focused on vibration analysis and modal analysis of mechanical systems and structures. He is also interested in the development of modern optical methods of experimental mechanics such as high-speed digital image correlation, electronic speckle pattern interferometry and laser vibrometry. He is co-author of 2 monographs, 3 university text books and more than 50 publications published in journals, conference proceedings at Slovakia and abroad. He participates in the solving a several grant projects and different tasks in cooperation with the practice.

Martin Hagara, Ing. Ph.D., (born in 1985) is a research worker at the Department of Applied Mechanics and Mechanical Engineering, Technical University of Košice, Slovakia. The research, he carries out, is focused on digital image correlation method and its implementation into solving problems of dynamics and stress/strain analysis. He is also interested in some others optical techniques such as electronic speckle pattern interferometry and laser vibrometry. In 2011 he attended a trimestral internship in Dantec Dynamics GmbH in UIm, Germany. He is the author and co-author of more than 40 publications published in journals, conference proceedings in Slovakia and abroad. He participates in the solving of several grant and state projects. 